\title{
Benefits of reviewing pancreatic function in children with cystic fibrosis
}

\author{
Rachael Marpole ${ }^{1}$ and Andrew Wilson ${ }^{1}$ \\ ${ }^{1}$ Perth Children's Hospital
}

February 6, 2022

\begin{abstract}
Background: Cystic fibrosis is a common cause of pancreatic exocrine insufficiency. Clinically pancreatic insufficiency is treated as a permanent problem. However, new cystic fibrosis transmembrane conductance regulator (CFTR) protein modulator therapies, like ivacaftor, can change pancreatic function in children. Also, children with residual CFTR function mutations may be pancreatic insufficient in infancy and spontaneously become pancreatic sufficient. Aims: To evaluate the benefit of reviewing pancreatic function, testing and diagnosis, of a cohort and repeat testing to patients with the potential to change function. Methods: all children with cystic fibrosis managed at Perth Children's Hospital at the end of 2019 were reviewed for pancreatic function testing at diagnosis, genotype and current modulator and pancreatic enzyme replacement therapy use. Children with the potential to change pancreatic function were offered repeat testing. Results: 204 children were included. 11 children were prescribed ivacaftor and four of these became pancreatic sufficient. Five children with a residual CFTR function mutation were pancreatic insufficient at diagnosis. Four became pancreatic sufficient. Conclusion: there is benefit in re-evaluating pancreatic function in children with cystic fibrosis regularly, especially if on ivacaftor or pancreatic insufficient with a residual CFTR function mutation.
\end{abstract}

\section{Benefits of reviewing pancreatic function in children with cystic fibrosis}

Rachael Marpole, MBBS 1,2,3

Andrew C Wilson, FRACP 1, 2, 3

\section{Affiliations}

1. Department of Respiratory and Sleep medicine, Perth Children's Hospital, Nedlands, WA, Australia

2. The University of Western Australia, Faculty of Medicine Dentistry and Health Sciences, Division of Paediatrics, Crawley, WA, Australia

3. Wal-Yan Respiratory Research Centre, Telethon Kids Institute, Nedlands, WA Australia

Funding : Rachael Marpole received funding from the WA Health Registrar Research Fellowship and Child and Adolescent Health Service Research Fellowship.

\section{Corresponding author}

Name: Dr. Rachael Marpole

Mailing address: Respiratory and Sleep Medicine Department, Perth Children's Hospital, 15 Hospital Avenue, Locked Bag 2010, Nedlands WA 6009, Australia.

Phone number: +61 864562222

Email: rachael.marpole@health.wa.gov.au 
ORCID Rachael Marpole https://orcid.org/0000-0002-2652-0560

Key words Cystic fibrosis, Pancreatic function, CFTR modulators, residual CFTR function, child, paediatric

Running head: Reviewing pancreatic function in $\mathrm{CF}$

To the Editor,

Cystic fibrosis $(\mathrm{CF})$ is a common autosomal recessive disease that causes pancreatic exocrine insufficiency (PI) in $90 \%$ of cases $^{1}$. Over the last decade new CF transmembrane conductance regulator (CFTR) protein modulator therapies, like ivacaftor, have become available. Ivacaftor can cause children with a gating mutation, up to 14 years of age, born with a meconium ileus to become pancreatic exocrine sufficient (PS) ${ }^{2}$. Of note, children with residual CFTR function mutations may be PI in infancy and then spontaneously become $\mathrm{PS}^{3}$.

Current recommendations from the Nutrition guidelines for CF in Australia and New Zealand suggest all children with CF have pancreatic function tested at diagnosis ${ }^{1}$. It is also suggested that repeat testing can help guide pancreatic enzymes replacement therapy (PERT) in patients on CF modulators. The aim of this study was to evaluate the benefit of reviewing pancreatic function testing and diagnosis of PS/PI of a cohort of CF patients and offering repeat testing to patients who had potential to become PS since initial testing.

Institutional ethics approval was granted prior to commencement of the study. All children with CF living in Western Australia were included. Retrospective review of the following included age, sex, birthplace, genotype, eligibility for and prescription of modulator therapy, current pancreatic function status, previous testing, meconium ileus at birth, and if prescribed PERT. After review, patients who had potential to become PS were offered testing prospectively.

There was 204 children and adolescents with CF being managed at Perth Children's Hospital the only tertiary paediatric hospital in Western Australia, at the end of 2019. $95(46 \%)$ were males, with an age range of 0-18 years. Exocrine pancreatic function had been tested in 177/204 (86\%), with the majority having faecal pancreatic elastase testing. Out of the tested cohort $73 \%$ were diagnosed as PI (130/177).

\section{Ivacaftor}

All 12 patients eligible for ivacaftor were pancreatic insufficient, 11 were on PERT (table 1). One patient ceased ivacaftor due to poor tolerance. All taking ivacaftor were offered repeat testing. 81\% (9/11) completed this. Four had become PS since starting ivacaftor (one clinically), with three stopping PERT. Two of the three patients experienced recurrent abdominal pain when taking PERT, which resolved on ceasing. Time on ivacaftor to stopping PERT varied from 14 months to 3 years. Age at stopping was 5, 9 and 10 years. One infant who tested initially as PI was not started on PERT due to lack of symptoms of malabsorption, became PS after six months of ivacaftor.

Other patients continue to be PI and on PERT despite being prescribed ivacaftor for up to 8 years. This includes an adolescent who developed pancreatitis twice after being on ivacaftor for 6 years. His pancreatic elastase has increased from $<15$ to $120 \mathrm{microg} / \mathrm{g}$.

\section{Residual CFTR function}

Of the 35 patients with at least one residual CFTR function mutation, 29 have had pancreatic elastase above $200 \mathrm{mcg} / \mathrm{g}$ since diagnosis and one had never been tested however was clinically PS. Two had previously become PS before the age of 2 years. The remaining three were offered retesting. All completed with two becoming PS at age 3 and 16 years. See table 2 .

In this study there were three clear findings. Firstly, treatment with ivacaftor is associated with a change from PI to PS in children with gating mutations. There are two multicentred studies that review pancreatic function in children with gating mutations on ivacaftor ${ }^{4,5}$. They include 12-24-month-olds and 2-5-yearolds. After 24 weeks of ivacaftor 18-30\% became PS. In our cohort 36\% (4/11) became PS after starting 
ivacaftor. Children are more likely to change function if initial pancreatic elastase is more than $50 \mathrm{microg} / \mathrm{g}$ and if ivacaftor is started at a younger age. Potentially children eligible for ivacaftor at one year of age with borderline pancreatic function can now be managed without PERT if growing well.

Secondly, children with PI and a residual CFTR function mutation can spontaneously become PS ${ }^{3}$. During this review,one patient with a residual CFTR function mutation continues to be PI. Most changed to PS prior to age 4 years and if their initial pancreatic elastase was over 150 micrograms/g.

Thirdly, new abdominal pain or constipation when taking PERT may be a sign of change in pancreatic function in children with the potential to change. 50\% (2/4) of the children on ivacaftor experienced pain with PERT and one child with a residual CFTR function mutation became constipated prior to repeat testing. Acknowledging that abdominal pain and constipation can be common in cystic fibrosis patients. The limitations of this study are it is from a single centre. There are small numbers. Also, some results are limited by missing data especially in children who had moved to Western Australia.

The future of CF care is rapidly changing given the advancement of modulator therapy. Recurrent abdominal pain and constipation may be caused by improvement in pancreatic function. Regular reassessment of pancreatic function will prevent inappropriate PERT prescription and reduce side effects. Future research in this area should be included in multicentre phase 3 trials on new modulator treatment, especially if involving young children who are PI.

In children with $\mathrm{CF}$, there is benefit to reviewing pancreatic function regularly, especially if on ivacaftor for a gating mutation, have residual CFTR function mutations or have new abdominal pain when taking PERT.

\section{References}

1. Saxby N, Painter C, Kench A, King S, Crowder T, Van der Haak N.Nutrition guidelines for cystic fibrosis in Australia and New Zealand. Sydney: Thoracic Society of Australia and New Zealand; 2017.

2. Nichols AL, Davies JC, Jones D, Carr SB. Restoration of exocrine pancreatic function in older children with cystic fibrosis on ivacaftor.Paediatr Respir Rev. 2020;35:99-102.

3. Poli P, Conforti C, Gennari E, Padoan R. Transitory pancreatic insufficiency in cystic fibrosis children. $J$ Cyst Fibros.2020;19(1):e5-e6.

4. Davies JC, Cunningham S, Harris WT, et al. Safety, pharmacokinetics, and pharmacodynamics of ivacaftor in patients aged $2-5$ years with cystic fibrosis and a CFTR gating mutation (KIWI): an open-label, single-arm study. The Lancet Respiratory Medicine.2016;4(2):107-115.

5. Rosenfeld M, Wainwright CE, Higgins M, et al. Ivacaftor treatment of cystic fibrosis in children aged 12 to $<24$ months and with a CFTR gating mutation (ARRIVAL): a phase 3 single-arm study. The Lancet Respiratory Medicine. 2018;6(7):545-553.

\section{Authors}

Rachael Marpole, MBBS 1, 2, 3

Andrew C Wilson, FRACP 1, 2, 3

\section{Affiliations}

1. Department of Respiratory and Sleep medicine, Perth Children's Hospital, Nedlands, WA, Australia

2. The University of Western Australia, Faculty of Medicine Dentistry and Health Sciences, Division of Paediatrics, Crawley, WA, Australia

3. Wal-Yan Respiratory Research Centre, Telethon Kids Institute, Nedlands, WA Australia

Funding : Rachael Marpole received funding from the WA Health Registrar Research Fellowship and Child and Adolescent Health Service Research Fellowship.

\section{Declarations}


Competing interests: None declared

\section{Corresponding author}

Name: Dr. Rachael Marpole

Mailing address: Respiratory and Sleep Medicine Department, Perth Children's Hospital, 15 Hospital Avenue, Locked Bag 2010, Nedlands WA 6009, Australia.

Phone number: +61 864562222

Email: rachael.marpole@health.wa.gov.au

ORCID Rachael Marpole https://orcid.org/0000-0002-2652-0560

\section{Hosted file}

Benefits of reviewing pancreatic function in children with cystic fibrosis tables.docx available at https://authorea.com/users/459083/articles/555465-benefits-of-reviewingpancreatic-function-in-children-with-cystic-fibrosis 